\title{
The Classificaton of EEG Signals Recorded in Drunk and Non-Drunk People
}

\author{
Ziya Ekşi \\ Sakarya University \\ Department of Electronics and \\ Computer Education
}

\author{
Akif Akgül \\ Sakarya University \\ Department of Electronics and \\ Computer Education
}

\author{
Mehmet Recep Bozkurt \\ Sakarya University \\ Department of Electrical and \\ Electronics Engineering
}

\begin{abstract}
Alcoholic beverages are widely used in many societies. People can maintain their everyday lives while using a certain amount of alcohol. However, when alcohol is used intensively, it prevents healthy thinking, undermines decision making and reflex functions. This decrease in reflexes brings increase in traffic and job accidents. In order to determine the capability of a person to do a job, the detection of alcohol level is important. Nowadays, breathalyzers are used for the purpose of such detection. These devices measure the amount of alcohol rather than loss of function caused by alcohol. But the amount of alcohol taken show different effects from person to person. In this study people were tested and determined whether they were alcoholic with the help of EEG data. Preprocessing was performed on the EEG data set before the process of detection, followed by the training of ANN (Artificial Neural Network) and its test. Based on the obtained best performance value, an interface was designed on MATLAB for users.
\end{abstract}

\section{General Terms}

Artificial Neural Networks, Biomedical Signal Processing

\section{Keywords}

EEG signals, Artificial Neural Network, Signal processing, Matlab Interface

\section{INTRODUCTION}

The excessive consumption of alcoholic beverages is an important social and medical problem. This problem causes adverse social and economic effects on the individual drinker, the drinker's immediate environment and society as a whole. Indeed, other people rather than the drinker can be affected, for example, by related traffic accidents or violence. Depending on the amount used, alcohol can reduce coordination, slow reflexes and lead to over-confidence. Alcohol also depresses parts of the central nervous system - it slows down some of brain functions. Nowadays, testing for alcohol consumption is done using a breathalyzer test. Ethyl alcohol can also be measured in blood sample, urine, or in saliva.

Figure 1 shows the detection of EEG signals from the human brain [1]. With the help of these signals, whether a person is drunk or not can be understood. In reality, the signals received from human brain contain information about the person's current status. The signals received from drunk people change in a certain range of frequency. Thus, alcohol's impact on human behavior can be detected much more clearly than using only breathalyzers.

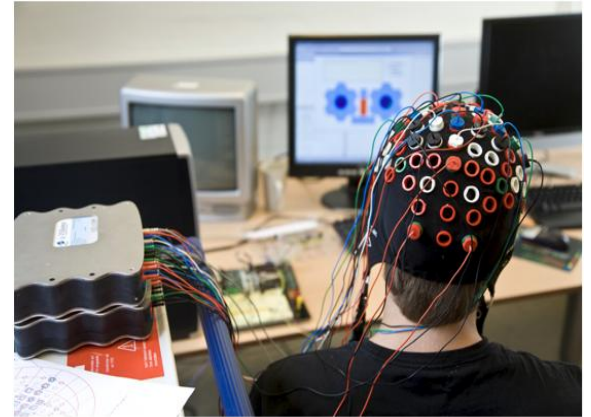

Fig 1: Measuring EEG signals [1]

Practically, the measurement of alcohol usage with EEG signals is not as easy as with breathalyzers, but today with spreading portable EEG devices, the measurements of the EEG signals can be made much easier and therefore much more accurate results can be obtained. There are various studies in the literature on EEG signals. For example, the study published by E. Malar et al. [2], proposes a new approach for detecting drunk drivers by the analysis of EEG spectral power density. Pradhan et al. [3] implements a two layered network for the analysis of EEG signals successfully. Jiang et al [4] classifies and examines EEG signal characteristics of Sleep Apnea Syndrome (SAS) by using wavelet transform and an ANN. Hazarika et al. [5] has benefited from EEG signals in order to make the classification of normal, schizophrenic and obsessive people using wavelet transform and three-layered feedforward network. Kiymık and et al proposes [6] a wavelet transform and ANN method for detecting alert, drowsy and sleep states. Bellotti et al. [7] classifies spontaneous EEG signals in migranie using feature extraction and supervised neural network. Puthankattil et al [8] classifies normal and depression patients using relative wavelet energy (RWE) and ANN according to EEG signals. Sabeti et al [9] performes EEG signal classification of schizophrenic and control participants using feature extraction. Olejarczyk et al [10] identifies the effects of different volatile anaesthetics on the spectro-temporal pattern of EEG. They perform spectral analysis of EEG signals using the discrete-time Fourier transform. Sahin et al [11] classifies the partial epilepsy groups using Multilayer Perceptron Neural Networks (MLPNNs) according to EEG signals.

In this study, a data set of EEG signals was used to design a system that detects whether a subject is drunk or non-drunk. The dataset contained drunk and non-drunk subjects' EEG signals. Pre-processing of the data was carried out by using Yule-Walker method, which is treated briefly in Section II. By this method, the data in the set are reduced by analyzing via parametric techniques, thus shortening the processing 
time. After that, the classification of drunk and non-drunk subject was done using ANN. For a better classification, three different epochs were implemented. According to the best training result, an interface on MATLAB was designed for potential users. The interface allows users to determine readily whether a subject is drunk or not.

In this paper, the nature of EEG signal, the definition of ANN and Yule-Walker method are treated in Section 2. Section 3 is assigned to experimental application and performance evaluation. And Section 4 includes conclusion and recommended future works.

\section{METHODOLOGY}

\subsection{Electroencephalography (EEG)}

Bioelectrical signals obtained as a result of brain neural activity are called EEG [12]. Human mental activities can be observed via EEG signals. EEG signals are recorded by electrodes placed over the head. The amplitude of these signals varies between 1-100 $\mu \mathrm{V}$ and their frequency spectrum lies between $0.5-100 \mathrm{~Hz}$. EEG signals are divided into 4 groups [13] according to their frequency contents as shown in table 1 .

Table 1. Frequency ranges of EEG signals

\begin{tabular}{|l|l|}
\hline Delta $(\boldsymbol{\delta})$ & $0.5-3 \mathrm{~Hz}$ \\
\hline Theta $(\boldsymbol{\theta})$ & $4-7 \mathrm{~Hz}$ \\
\hline Alpha $(\boldsymbol{\alpha})$ & $8-13 \mathrm{~Hz}$ \\
\hline Beta $(\boldsymbol{\beta})$ & $>13 \mathrm{~Hz}$ \\
\hline
\end{tabular}

These frequency values vary depending on people's instant mental attitudes. Alpha waves are observed in normal, calm people. The amplitude value of these waves is approximately $50 \mu \mathrm{V}$. Beta waves occur usually in cases of stress. Theta waves are seen in children, and adults when they are disappointed and tensioned. Delta waves are observed in infants and in cases of severe organic brain diseases [13]. In Figure 2, EEG waves with their four rhythms are shown [14].

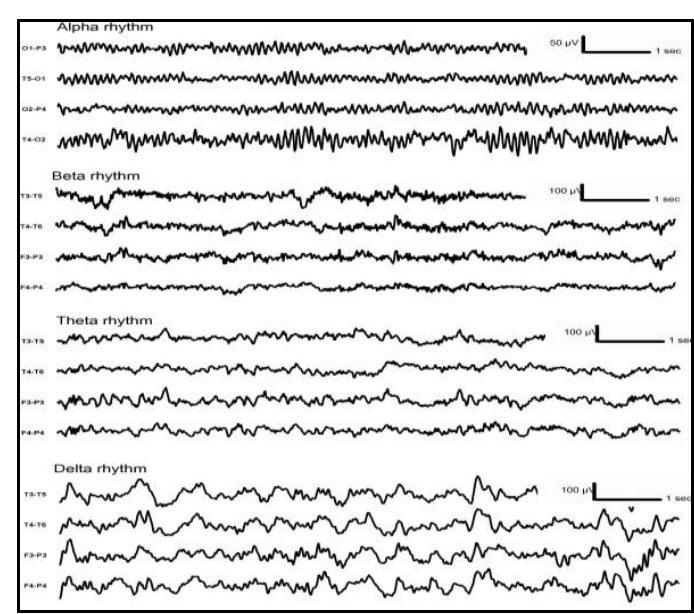

Fig 2: EEG waves with their four rhythms [14]

\subsection{Artificial Neural Network (ANN)}

ANN is developed on the model of human biological nervous system. ANN is a mathematical calculation method based on learning and making decisions [15]. It is a computer based system which is used to solve difficult and complex problems. A comparison of artificial neural network and biological neural network is given in Table 2 [16].
Table 2. A Comparison of Biological and Artificial Neural Network

\begin{tabular}{|l|l|}
\hline Biological Neural Network & Artificial Neural Network \\
\hline Neuron & Processing element \\
\hline Synapse & Weight \\
\hline Dendrite & Add Function \\
\hline Cell Body & Transfer/Activation Function \\
\hline Axon & Exit \\
\hline
\end{tabular}

ANN is categorized as feedforward and feedback according to their architectural structures. Similarly, ANN is classified into three groups with respect to learning approachs, namely supervised, unsupervised and reinforcement learning. Hebb, Hopfield, Kahonen and Delta are used as learning rules [17].

In this paper, feedforward error backpropagation ANN (FEBANN) is used. This ANN type is preferred due to the ease of implementation, higher speed and minor training sets requirement.

\subsection{YULE-WALKER Method}

This method is called autocorrelation in the some sources. Autocorrelation equations are available on the basis of Yule-Walker which is used for Autoregressive (AR) model, so these two names are used interchangeably in the literature [15]. In this method, AR coefficients ap $(\mathrm{k})$ are calculated using autocorrelation equations.

$\left[\begin{array}{ccccc}r_{x}(0) & r_{x} *(1) & r_{x}^{*}(2) & \cdots & r_{x}^{*}(p) \\ r_{x}(1) & r_{x}(0) & r_{x} *(1) & \cdots & r_{x}^{*}(p-1) \\ r_{x}(2) & r_{x}(1) & r_{x}(0) & \cdots & r_{x} *(p-2) \\ \vdots & \vdots & \vdots & & \vdots \\ r_{x}(p) & r_{x}(p-1) & r_{x}(p-2) & \cdots & r_{x}(0)\end{array}\right]\left[\begin{array}{c}1 \\ a_{p}(1) \\ a_{p}(2) \\ \vdots \\ a_{p}(p)\end{array}\right]=\varepsilon_{p}\left[\begin{array}{c}1 \\ 0 \\ 0 \\ \vdots \\ 0\end{array}\right]$

Here,

$$
r_{x}(k)=\frac{1}{N} \sum_{n=0}^{N-1-k} x(n+k) x *(n) ; k=0,1, \ldots . p
$$

If the matrix equation above is solved for $\mathrm{ap}(\mathrm{k})$, then

$$
|b(0)|^{2}=\varepsilon_{p}=r_{X}(0)+\sum_{k=1}^{p} a p(k) r_{x} *(k)
$$

is obtained.

As a result of this equality, it will be possible to make a prediction about the power spectrum [18].

\section{EXPERIMENTAL WORK AND PERFORMANCE EVALUATION}

A block diagram for experimental work procedure is provided in Figure 3. 


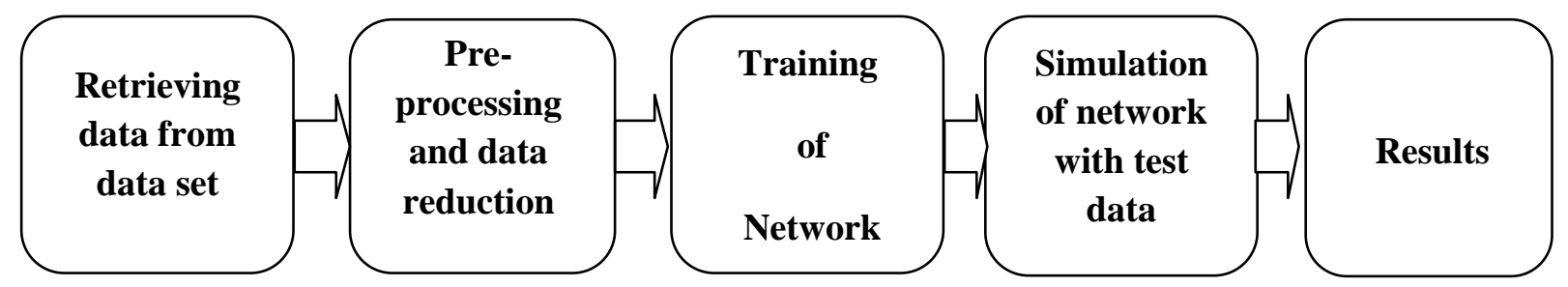

Fig 3: Block Diagram of the Experimental Work

The first stage in the block diagram is data retrieval. In this stage, drunk and non-drunk subjects EEG data were used. In this stage, two different data files containing 256 EEG for each of 50 drunk subjects and 256 EEG for each of 50 non-drunk subjects were retrieved from Donald Bren School of Information and Computer Sciences [19]. Among these, 40 drunk subjects and 40 non-drunk subjects EEG data were used to train ANN. The remaining data were used in order to test the network. In the second stage, pre-processing and data reduction were realized using Yule-Walker method on MATLAB platform.

The number of EEG data for both drunk and non-drunk subjects' data were reduced from 256 to 8 by Yule-Walker method. In the third stage, the data were presented to the network and it was trained. The training used 40 drunk and 40 non-drunk subjects' EEG data. Then, test data were fed to the trained network. The remaining 10 drunk and 10 non-drunk subjects' data were used as test data.

In the simulation test of the network 300,900 and 1500 epochs were performed. The obtained results were $80 \%$, $95 \%$ and $80 \%$ success, respectively. With respect to the percentage success, the user interface was designed.

In the study, the best performance was reached in 900 epochs. Simulation results obtained with 900 epochs are given in Figure 4, where level 0 represents non-drunk, level 1 drunk and level 2 indefinite. As Figure 4 illustrates, only 1 out of 20 subjects is undetermined. This instable data shows indefiniteness.

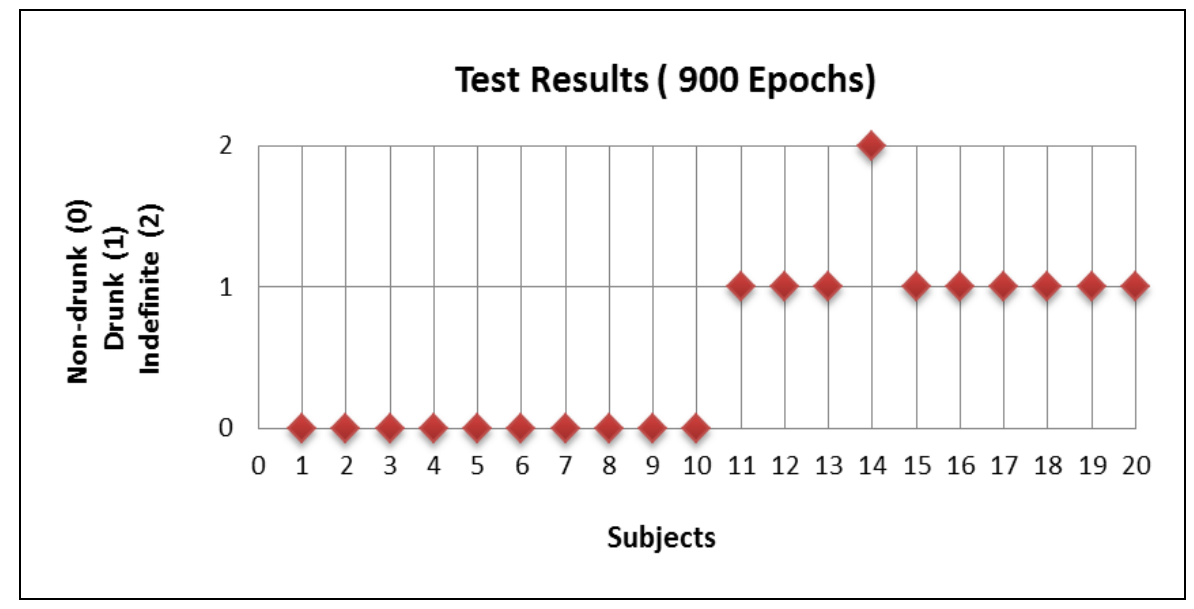

Fig 4: The output of the neural network simulation for 900 epochs

In Figure 5, performance percentage of the network is shown success is $80 \%$, with $95 \%$ for 900 epochs, which is the best. or 300,900 and 1500 epochs. For 300 and 1500 epochs,

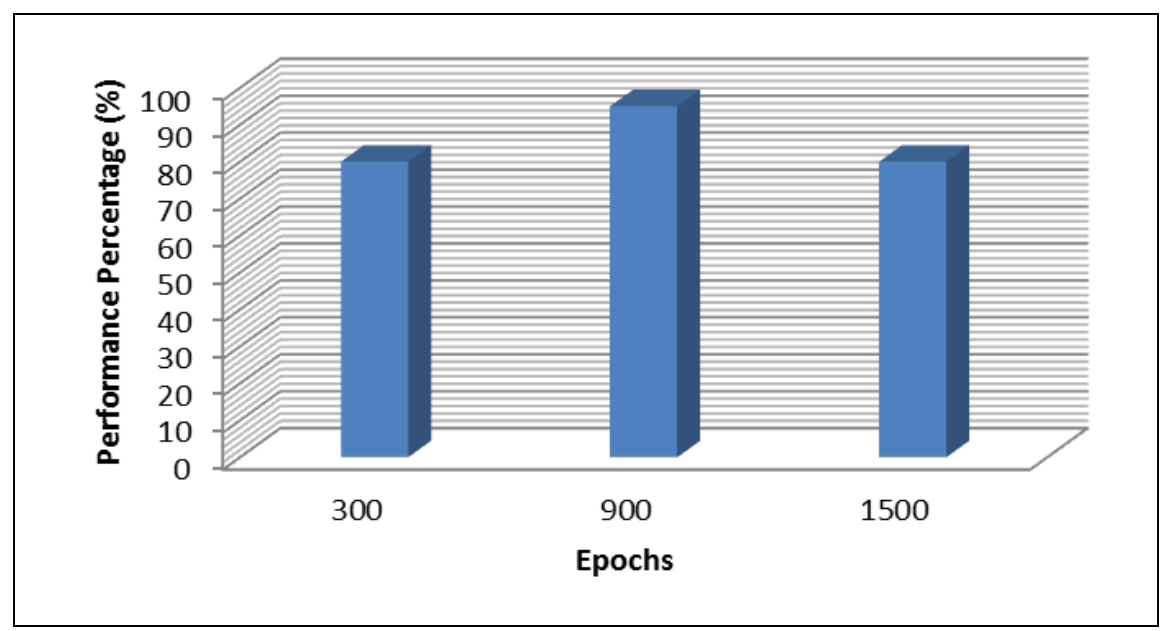

Fig 5: Network performance for different values of epochs 
In the interface study for the network, the best performance epoch were used. The interface consists of file path, data screen and test part. When the full path of data files are entered, data is transferred from the recorded file to the interface path with OK button. In Figure 6, the interface screen including test data is shown. In the designed interface, numbers $1,2,3 \ldots$ in columns denote subjects, and numbers $1,2,3 \ldots$ in lines show EEG data taken at different times from the subjects. By pressing the "DO TEST" button whether the subject is drunk or not can be determined. For example, according to test results provided in Figure 6, the subject numbered 4 is determined as non-drunk and the subject numbered 18 is determined as drunk.

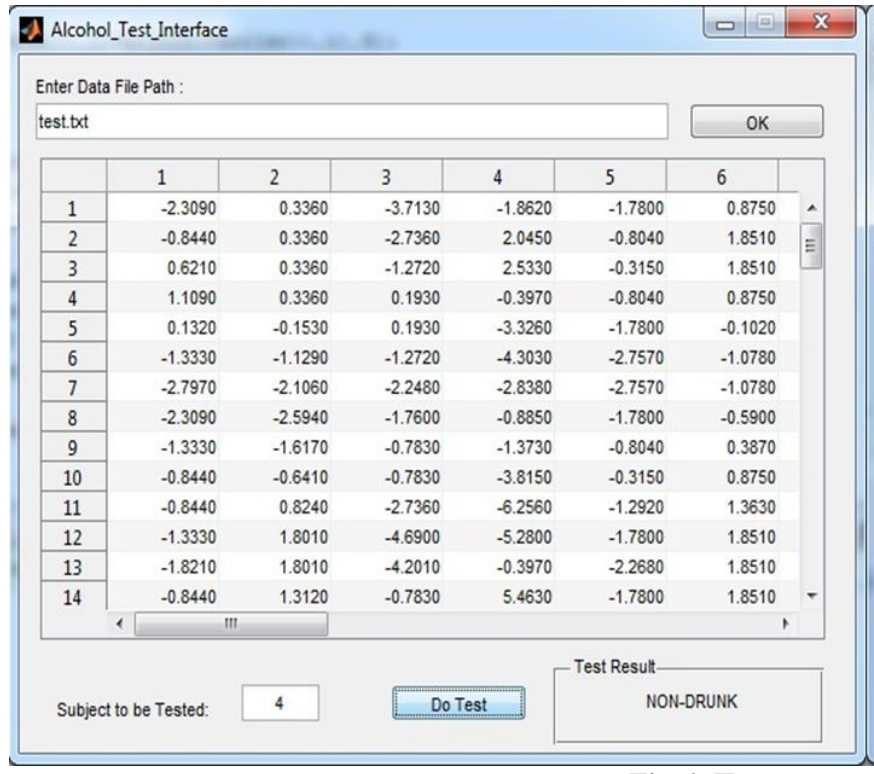

\begin{tabular}{|c|c|c|c|c|c|c|c|}
\hline \multicolumn{6}{|c|}{ Alcohol_Test_Interface } & \multicolumn{2}{|c|}{\begin{tabular}{|l|l|l}
0 & 0 & $x$ \\
\end{tabular}} \\
\hline \multicolumn{8}{|c|}{ Enter Data File Path : } \\
\hline \multicolumn{6}{|l|}{ test.txt } & \multicolumn{2}{|c|}{$\mathrm{OK}$} \\
\hline & 1 & 2 & 3 & 4 & 5 & 6 & \\
\hline 1 & -2.3090 & 0.3360 & -3.7130 & -1.8620 & -1.7800 & 0.8750 & $A$ \\
\hline 2 & -0.8440 & 0.3360 & -2.7360 & 2.0450 & -0.8040 & 1.8510 & E \\
\hline 3 & 0.6210 & 0.3360 & -1.2720 & 2.5330 & -0.3150 & 1.8510 & L \\
\hline 4 & 1.1090 & 0.3360 & 0.1930 & -0.3970 & -0.8040 & 0.8750 & \\
\hline 5 & 0.1320 & -0.1530 & 0.1930 & -3.3260 & -1.7800 & -0.1020 & \\
\hline 6 & -1.3330 & -1.1290 & -1.2720 & -4.3030 & -2.7570 & -1.0780 & \\
\hline 7 & -2.7970 & -2.1060 & -2.2480 & -2.8380 & -2.7570 & -1.0780 & \\
\hline 8 & -2.3090 & -2.5940 & -1.7600 & -0.8850 & -1.7800 & -0.5900 & \\
\hline 9 & -1.3330 & -1.6170 & -0.7830 & -1.3730 & -0.8040 & 0.3870 & \\
\hline 10 & -0.8440 & -0.6410 & -0.7830 & -3.8150 & -0.3150 & 0.8750 & \\
\hline 11 & -0.8440 & 0.8240 & -2.7360 & -6.2560 & -1.2920 & 1.3630 & \\
\hline 12 & -1.3330 & 1.8010 & -4.6900 & -5.2800 & -1.7800 & 1.8510 & \\
\hline 13 & -1.8210 & 1.8010 & -4.2010 & -0.3970 & -2.2680 & 1.8510 & \\
\hline \multirow[t]{2}{*}{14} & -0.8440 & 1.3120 & -0.7830 & 5.4630 & -1.7800 & 1.8510 & - \\
\hline & $1 \square$ & 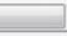 & & & & & \\
\hline \multirow{2}{*}{\multicolumn{2}{|c|}{ Subject to be Tested: }} & & & & Resul: & & \\
\hline & & 18 & $D_{0}$ & & \multicolumn{2}{|c|}{ DRUNK } & \\
\hline
\end{tabular}

Fig 6: Test screen of the interface

\section{CONCLUSION}

In this study, an alternative method was presented for determining alcohol usage. The classification of EEG signals recorded from drunk and non-drunk subjects was implemented successfully. The designed system consists of a pre-processing unit, an ANN and a user interface. Yule-Walker power spectral densities were used in the preprocessor, in which each EEG signal frame consisting of 256 samples yielded 8 inputs to the ANN after reduction. In the ANN, the best result was obtained in 900 epochs with $95 \%$ success rate using the reduced data. The interface was based on the parameters providing the best performance. Subjects' mental conditions were determined using these parameters in the interface. The determination of a subject's situation as non-drunk or drunk can be easily done by loading the test data to the interface. It was observed that the simulation performance can be improved by increasing the training data set.

\section{REFERENCES}

[1] Figure of measuring EEG signals, http://bmm.etu.edu.tr/tr/content/biyoelektronik-sinyalisleme, Last accessed 20.03.2012.

[2] Malar E., Gauthaam M., Chakravarthy D., 2011, “A Novel Approach for the Detection of Drunken Driving using the Power Spectral Density Analysis of EEG", International Journal of Computer Applications (0975 8887), Volume 21- No.7, pp.10-14.

[3] Pradhan N., Sadasivan P. K, Arunodaya G. R., 1996, "Detection of Seizure Activity in EEG by An Artificial Neural Network: A Preliminary Study", Computers and Biomedical Research, No.29, pp. 303-313.
[4] Lin R., Lee R., Tseng C., Zhou H., Chao C., Jiang J., “A New Approach for Identifying Sleep Apnea Syndrome using Wavelet Transform and Neural Networks", Biomedical Engineering: Applications, Basis and Communications, 2006, Vol. 18, pp. $138-143$.

[5] Hazarika N., Chen J. Z., Tsoi A. C., Sergejew A., 1997, "Classification of EEG Signals Using the Wavelet Transform”, Signal Processing, Vol.59, No.1, pp. 61-72.

[6] Kıymık M. K., Akın M., Subası A., 2004, "Automatic Recognition of Alertness Level by Using Wavelet Transform and Artificial Neural Network", Journal of euroscience Methods, vol. 139, pp. 231-240.

[7] Bellotti R., De Carlo F., Tommaso M. D, Lucente M., 2007, "Classification of Spontaneous EEG Signals in Migraine", Physica A: Statistical Mechanics and its Applications, vol. 382, Issue 2, pp. 549-556.

[8] Puthankattil S.D., Joseph P.K., "Classification of EEG Signals in Normal and Depression Conditions by ANN Using RWE and Signal Entropy", Journal of Mechanics in Medicine and Biology, 2012, Vol. 12, No. 4, pp. 1-13.

[9] Sabeti M., Katebi S. D., Boostani R., Price G.W., 2011, "A New Approach for EEG Signal Classification of Schizophrenic and Control Participants", Expert Systems with Applications, vol. 38, pp. 2063-2071.

[10] Olejarczyk E., Sobieszek A., Runder R., Marciniak R., Wartak M., Stasiowski M., Jalowiecki P., 2010, "Spectral analysis of the EEG-signal Registered during Anaesthesia Induced by Propofol and Maintained by 
Fluorinated Inhalation Anaesthetics", Biocybernetics and Biomedical Engineering, vol. 30, pp. 55-70.

[11] Şahin C., Ogulata S.N., Aslan K., Bozdemir H., Erol R., "A Neural Network-Based Classification Model for Partial Epilesy by EEG Signals", International Journal of Pattern Recognition and Artificial Intelligence, 2008, Vol. 22, No. 5, pp. 973-985.

[12] Batar H., 2007, "Analysis Of EEG Signals Using The Wavelet Transform And Artificial Neural Network", M.Sc. Thesis, Süleyman Demirel University Graduate School of Applied and Natural Sciences, Isparta, Turkey.

[13] Yazgan E., Korürek M., 1995. “Tıp Elektroniği”, İstanbul, Turkey.

[14] Tyvaert L.,Van P.L., Grova C., Dubeau F., Gotman J., 2008, "Effects of Fluctuating Physiological Rhythms
During Prolonged EEG-fMRI Studies" Clinical Neurophysiology 119, pp. 2762-2774.

[15] Bozkurt M. R., 2007, "Pre-Processing And Classification Of EMG Signals By Using Modern Methods", $\mathrm{PhD}$ Thesis, Sakarya University Graduate School of Applied and Natural Sciences, Sakarya, Turkey.

[16] Vatansever F., Doğalı G., 2011, “Klasik Enterpolasyon Yöntemleri ve Yapay Sinir Ağı Yaklaşımlarının Karşıllaştırılması", 6th International Advanced Technologies Symposium (IATS'11), pp. 51-54.

[17] Elmas Ç., 2003, Yapay Sinir Ağları, Seçkin Yayıncılık, Ankara.

[18] Hayes M.H, 1996 , "Statistical Digital Signal Processing and Modelling", New York: John Wiley and Sons.

[19] http://archive.ics.uci.edu/ml/databases/eeg/eeg_full/ 